\title{
Human Papillomavirus Type 16
}

National Cancer Institute

\section{Source}

National Cancer Institute. Human Papillomavirus Type 16. NCI Thesaurus. Code C14338.

A virus comprised of a protein coat (capsid) surrounding a circular, double-stranded DNA organized into coding and non-coding regions, which can induce skin and mucosal epithelial lesions. Human papillomavirus-16 (HPV16) has been directly linked to cervical cancer and is significantly associated with invasiveness. Progression from low- to highgrade neoplasia is often associated with the integration of the HPV16 genome into the host chromosome. 